\title{
Preliminary study of the Thai-version of the Scale for the Assessment of Positive Symptoms (SAPS-Thai): content validity, known-group validity, and internal consistency reliability
}

\author{
ThammanaRd CHARERNBOON ${ }^{1}$ \\ 1 Department of Psychiatry and Department of Clinical Epidemiology, Faculty of Medicine, Thammasat University, Pathumthani, Thailand. \\ Institution where the study was conducted: Faculty of Medicine, Thammasat University Hospital, Pathumthani, Thailand.
}

Received: 7/18/2018 - Accepted: 12/9/2018

DOl: 10.1590/0101-60830000000183

\begin{abstract}
Objective: To assess the psychometric properties of the Thai version of the Scale for the Assessment of Positive Symptoms (SAPS-Thai). Methods: Content validity was evaluated by four psychiatrists who rated the SAPS-Thai, and the content validity indexes (CVI) were also analyzed. Known-group validity was assessed by comparing the SAPS-Thai score among thirty outpatients and ten inpatients with schizophrenia. Internal consistency was calculated using the Cronbach's coefficient alpha. Results: SAPS-Thai has excellent content validity, with an average-CVI of 0.92 . The inpatient group had a significant higher score for both the global SAPS-Thai scores [7.4 (1.95) vs. 1.93 (1.59), $\mathrm{p}<0.001$ ] and total SAPS-Thai scores [21.2 (11.8) vs. $3.67(2.87), \mathrm{p}<0.001]$ indicating good known-group validity. SAPS-Thai had a good internal consistency with a Cronbach's alpha of 0.87 . Discussion: SAPS-Thai provides a comprehensive measurement of positive symptoms and indicated content and known-group validity and a satisfactory level of internal consistency reliability.
\end{abstract}

Charernboon T / Arch Clin Psychiatry. 2019;46(1):5-8

Keywords: Positive symptoms, psychopathology, rating scale, schizophrenia.

\section{Introduction}

Schizophrenia is a complex chronic disease that causes impairment to occupational and social functions. Schizophrenia symptoms can be divided into positive and negative symptoms. Positive symptoms are thoughts and behaviors that are not normally present. The Diagnostic and Statistical Manual of Mental Disorders, 5th edition (DSM-5) describes four characteristics of positive symptoms, namely, delusions, hallucinations, disorganized speech, and disorganized behavior ${ }^{1}$.

For both clinical and research purposes, it is essential to have a standard, validated, and reliable assessment of the symptoms. The Scale for the Assessment of Positive Symptoms (SAPS) is one of the most widely used positive symptom rating scales used in clinical settings and clinical trials. It was developed by Andreasen (1984) specifically to assess positive symptoms in schizophrenia ${ }^{2}$. SAPS has been translated into many languages (for example, Chinese ${ }^{3}$ and Spanish ${ }^{4}$ ) but not Thai. Therefore, a Thai version of SAPS needs to be developed. Moreover, when translating a tool into a different language and culture, effective translation and validation studies of the measurement system must take precedence. For example, a few items were added to the Chinese version of SAPS, while several symptom definitions were changed during the translation process. The Chinese version demonstrated good internal consistency $(0.84)$ and an excellent inter-rater reliability $(0.95)$ for the overall score. It strongly correlated with the Brief Psychiatric Rating Scale 3 .

The objective of the present study was to develop a Thai version of SAPS to be available and examine its validity and reliability.

\section{Methods}

The study was comprised of two parts: the first part was the development of the Thai-SAPS and an examination of the content validity, while the second part was the assessment of its internal consistency and known-group validity.

\section{Thai version of the Scale for the Assessment of Positive Symptoms (SAPS-Thai)}

SAPS consist of thirty items and is divided into four domains: hallucinations, delusions, bizarre behavior, and formal thought disorder. Each item is rated on a six-point scale ranging from 0 to 5 and includes a global rating scale which is the summarized score of the symptoms in that domain. The total SAPS score can be calculated by totaling the scores of all thirty items. The global SAPS score is calculated by summarizing the score of each global rating scale. The total SAPS score ranges from $0-150$ and the global SAPS score of $0-20$. Higher scores indicate greater symptom severity ${ }^{2}$.

\section{Translation and cross-cultural adaptation}

Cross-cultural translation and adaptation of a questionnaire for use in a new language requires the use of a specific method to attain equivalence between the original and translated versions. The items must not only be translated well linguistically, but also must be culturally adapted to maintain the content validity of the measures ${ }^{5}$.

The Thai version of SAPS (SAPS-Thai) was developed using a forward and backward translation method ${ }^{5,6}$. In the forward translation, two psychiatrists (TC and TL) translated the original SAPS into Thai. After that, the two translators synthesized the translation results and the completed forward translation was conducted. During this process, small numbers of the symptom examples, i.e. incoherence and clanging, needed to be changed because they couldn't be literally translated with the exact meaning as the original English version. Therefore, the two translators created examples appropriate for the Thai language. The forward version was then back-translated into English by a professional English translator, with the discrepancies discussed to resolve the translation differences.

After the translation process, an expert committee consisting of four psychiatrists trialled the prefinal version of the questionnaire and gave comments. Later, all the experts rated the content validity 
of the final version. The content validity method and results are illustrated in the following topic and in Table 2. At this stage, it was decided that a few more symptom examples should be culturally adapted, while all the definitions and questions were similar to the original English version. For example, for grandiose delusions, " $\mathrm{He}$ may think he is actually some famous personage, such as Napoleon, or Christ", 'Napoleon' was changed to an ancient Thai King, while 'Christ' was changed to 'Buddha' due to the original personages being unfamiliar in Thai culture. For religious delusions, an example concerning Christian beliefs, "... beliefs about the Antichrist", was changed to symptoms more related to Buddhism. All the SAPS-Thai adaptations are summarized in Table 1.

A pilot study of the final version was conducted with three people with schizophrenia and two normal controls. There were no further modifications at this point. SAPS-Thai was then evaluated in the second part of the study.

\section{Content validity}

To evaluate content validity, Lynn (1986) suggested a minimum of three experts ; therefore, in this study, a panel of four certified psychiatrists were asked to rate each SAPS-Thai item and provide comments.

Each item rating was on a four-point scale (0-3). Two types of content validity were calculated. The first type was the item-level content validity index (item-CVI) for each SAPS-Thai item, with the I-CVI calculated as the number of experts giving a rating of 2 or 3 divided by the total number of experts. The second type of content validity was the average proportion of items rated as 2 or 3 across the experts (average-CVI) ${ }^{8}$. The content validity results are illustrated in Table 2.

\section{Internal consistency and known-group validity}

The second part of the study was a part of a larger study, entitled "Social Cognition in Schizophrenia Study". The main study was approved by the Ethics Committee of the Faculty of Medicine, Thammasat University (protocol number: MTU-EC-ES-6-043/59).

The participants included forty schizophrenia patients registered at Thammasat University Hospital, Thailand from $1^{\text {st }}$ May 2016 to $31^{\text {st }}$ December 2016. The participants were drawn from two sources: ten from the psychiatric inpatient unit and thirty from the psychiatric outpatient department. They were aged between 20 and 60 years old and had been clinically diagnosed with DSM-5 criteria of schizophrenia by certified psychiatrists. Exclusion criteria included those with a history of substance dependence (nicotine excluded), intellectual disability, and major neurological disorders.

\section{Statistical analysis}

Internal consistency was calculated by the Cronbach's coefficient alpha. Known-group validity was calculated by comparing the global SAPS and total SAPS score between the inpatient and outpatient groups using the Mann-Whitney $\mathrm{U}$ test. The inpatient group was expected to have a higher scores than the outpatients. A p-value of less than 0.05 indicated a statistical significance.

\section{Results}

The participants consisted of forty schizophrenia patients: ten inpatients and thirty outpatients. The majority of them were female $(55 \%)$ and their mean age was 35.9 years (SD 11.9) (Table 3).

\section{Content validity}

The results showed that SAPS-Thai had an excellent content validity with an average-CVI of 0.92 . The proportion of relevant of each expert ranged from 0.87-1.0. Every item except religious delusions had an item-CVI of at least 0.75 , while religious delusions had an item-CVI of 0.5 (Table 2).

\section{Known-group validity}

Known-group validity was assessed by comparing the SAPS-Thai score between inpatients and outpatients. The results demonstrated that the inpatient group had a significantly higher mean global SAPS score: 7.4 (1.95) vs. 1.93 (1.59), $\mathrm{p}<0.001$ and total SAPS score: 21.2 (11.8) vs. 3.67 (2.87), $\mathrm{p}<0.001$ (Table 3 ).

\section{Internal consistency reliability}

The Cronbach's alpha value demonstrated that SAPS-Thai had a good internal consistency (0.87).

Table 1. Adapted items of the Thai version of the Scale for the Assessment of Positive Symptoms (SAPS-Thai)

\begin{tabular}{|c|c|c|}
\hline Item & Original & Adaptation \\
\hline Grandiose delusions & $\begin{array}{l}\text { He may think he is actually some famous personage, such as a rock } \\
\text { star, Napoleon, or Christ. }\end{array}$ & $\begin{array}{l}\text { He may think is actually some famous personage, such as rock star, } \\
\text { ancient Thai King, or Buddha. }\end{array}$ \\
\hline Religious delusions & $\begin{array}{l}\text { The subject is preoccupied with false beliefs of a religious nature. } \\
\text { Sometimes these exist within the context of a conventional } \\
\text { religious system, such as beliefs about the Second Coming, the } \\
\text { Antichrist, or possession by the Devil. At other times, they may } \\
\text { involve an entirely new religious system or a pastiche of beliefs } \\
\text { from a variety of religions, particularly Eastern religions, such as } \\
\text { ideas about reincarnation or Nirvana. }\end{array}$ & $\begin{array}{l}\text { The subject is preoccupied with false beliefs of a religious nature. } \\
\text { Sometimes these exist within the context of a conventional } \\
\text { religious system, such as beliefs about reincarnation, Nirvana } \\
\text { or possession by the ghost. At other times, they may involve an } \\
\text { entirely new religious system or a pastiche of beliefs from a variety } \\
\text { of religions, particularly Western religions, such as ideas about the } \\
\text { Second Coming or the Antichrist. }\end{array}$ \\
\hline Incoherence & $\begin{array}{l}\text { Subject: "They're destroying too many cattle and oil just to make } \\
\text { soap. If we need soap when you can jump into a pool of water, and } \\
\text { then when you go to buy your gasoline, my folks always thought } \\
\text { they should, get pop but the best thing to get, is motor oil, and, } \\
\text { money. May, may as well go there and, trade in some, pop caps } \\
\text { and, uh, tires, and tractors to group, car garages, so they can pull } \\
\text { cars away from wrecks, is what I believed in". } \\
\text { None }\end{array}$ & Replaced with the example of incoherence in Thai. \\
\hline Clanging & $\begin{array}{l}\text { I'm not trying to make a noise. I'm trying to make sense. If you can } \\
\text { make sense out of nonsense, well, have fun. I'm trying to make } \\
\text { sense out of sense. I'm not making sense (cents) anymore. I have to } \\
\text { make dollars". } \\
\text { None }\end{array}$ & Replaced with the example of clang associations in Thai. \\
\hline
\end{tabular}


Table 2. Content validity of the Thai version of the Scale for the Assessment of Positive Symptoms

\begin{tabular}{|c|c|c|c|c|c|}
\hline Items & Expert 1 & Expert 2 & Expert3 & Expert 4 & Item CVI \\
\hline 1. Auditory hallucinations & $x$ & $x$ & $x$ & $x$ & 1 \\
\hline 2. Voices commenting & $x$ & $x$ & $x$ & $x$ & 1 \\
\hline 3. Voices conversing & $x$ & $X$ & $X$ & $x$ & 1 \\
\hline 4. Somatic or tactile hallucinations & $x$ & - & $X$ & $x$ & 0.75 \\
\hline 5. Olfactory hallucinations & $x$ & $x$ & $x$ & $x$ & 1 \\
\hline 6. Visual hallucinations & $x$ & $x$ & $x$ & $x$ & 1 \\
\hline 7. Persecutory delusions & $x$ & $x$ & $x$ & $x$ & 1 \\
\hline 8. Delusions of jealousy & $x$ & $x$ & $x$ & $x$ & 1 \\
\hline 9. Delusions of guilt or sin & $x$ & $x$ & $x$ & $x$ & 1 \\
\hline 10. Grandiose delusions & $x$ & - & $X$ & $x$ & 0.75 \\
\hline 11. Religious delusions & $x$ & - & $x$ & - & 0.5 \\
\hline 12. Somatic delusions & - & $x$ & $x$ & $x$ & 0.75 \\
\hline 13. Delusions of reference & $x$ & $x$ & $X$ & $x$ & 1 \\
\hline 14. Delusions of being controlled & $x$ & $x$ & $X$ & - & 0.75 \\
\hline 15. Delusions of mind reading & $x$ & $x$ & $x$ & $x$ & 1 \\
\hline 16. Thought broadcasting & $x$ & $x$ & $x$ & $x$ & 1 \\
\hline 17. Thought insertion & $x$ & $x$ & $x$ & $x$ & 1 \\
\hline 18. Thought withdrawal & $x$ & $x$ & $x$ & $x$ & 1 \\
\hline 19. Clothing and appearance & - & $x$ & $x$ & $x$ & 0.75 \\
\hline 20. Social and sexual behavior & $x$ & $x$ & $x$ & $x$ & 1 \\
\hline 21. Aggressive and agitated behavior & $x$ & $x$ & $x$ & $x$ & 1 \\
\hline 22. Repetitive or stereotyped behavior & $x$ & - & $x$ & $x$ & 0.75 \\
\hline 23. Derailment & $x$ & $x$ & $x$ & $x$ & 1 \\
\hline 24. Tangentially & $x$ & $x$ & $X$ & $x$ & 1 \\
\hline 25. Incoherence & $x$ & $x$ & $x$ & $x$ & 1 \\
\hline 26. Illogically & $x$ & $x$ & $x$ & - & 0.75 \\
\hline 27. Circumstantiality & $x$ & $x$ & $X$ & $x$ & 1 \\
\hline 28. Pressure of speech & $x$ & $x$ & $x$ & $x$ & 1 \\
\hline 29. Distractible speech & $x$ & $x$ & $x$ & $x$ & 1 \\
\hline 30. Clanging & $x$ & $x$ & $x$ & - & 0.75 \\
\hline Proportion relevant & 0.93 & 0.87 & 1 & 0.87 & $0.92^{*}$ \\
\hline
\end{tabular}

X: relevant; -: not relevant. * Average-content validity index.

Table 3. Characteristics and known-group validity

\begin{tabular}{|l|c|c|c|}
\hline & Inpatients $(\mathrm{n}=10)$ & Outpatients $(\mathrm{n}=30)$ & $\mathrm{p}$-value \\
\hline Gender: male (\%) & $5(50 \%)$ & $13(43.3)$ & $0.714^{*}$ \\
\hline Age: mean (SD) & $34.8(10.5)$ & $36.2(12.5)$ & $0.755^{* *}$ \\
\hline Global SAPS score: mean (SD) & $7.4(1.95)$ & $1.93(1.59)$ & $<0.001^{* *}$ \\
\hline Total SAPS score: mean (SD) & $21.2(11.88)$ & $3.67(2.87)$ & $<0.001^{* *}$ \\
\hline
\end{tabular}

${ }^{*}$ Chi-square. ${ }^{* *}$ Mann-Whitney $U$ test. SAPS: The Scale for the Assessment of Positive Symptoms.

\section{Discussion}

When translated and adapting Western tools to non-Western cultures there is an intrinsic tension between trying to either maintain the content as similar as possible to the original version or modifying it sufficiently to maximize its clinical utility in the target language. For the Thai version of the SAPS, the concept and definition of each SAPS item were similar to the original English version. Only minor adaptations were made to some of the symptom examples. Therefore, the authors believe that SAPS-Thai is comparable to the original English version and contains the same number of items and concepts. The present study demonstrated that SAPS-Thai had a good level internal consistency, content and known-group validity.

SAPS-Thai was confirmed to have an excellent content validity with an average-CVI of 0.92 . The only low item-CVI score was for 'religious delusions,' Which may be due to cultural differences. During the forward and backward translation process, this item was difficult for Thai people to understand when attempting to keep the translation as close to the original English text as possible. This is because most of the examples are related to Christianity, which is little known about in Thailand which is a majority Buddhist country. This led to a quite low 'religious delusions' item-CVI; therefore, in the final version, minor adaptations were made for this item to be more relevant to Thai culture.

While the validity and reliability of the Scale for the Assessment of Negative Symptoms (SANS) are frequently reported 10,11 , it quite surprising that the psychometric properties of SAPS are rarely reported. The participants in this study were divided into inpatients and outpatients to demonstrate known-group validity. The results showed that SAPS-Thai can discriminate between inpatients, who have more severe psychotic symptoms, from outpatients who generally have fewer symptoms. Both the global SAPS and total SAPS scores were significantly higher in the inpatient group. 
SAPS-Thai had a high internal consistency with a Cronbach's alpha of 0.87, which was slightly higher than Andreasen's $(1984,1990)$ original study $(0.65)^{2,12}$ but similar to the Chinese SAPS validation study $(0.84)^{3}$. Traditionally, an internal consistency greater than 0.8 is a desirable level for research tools, while over 0.9 is too high and most likely indicates unnecessary redundancy ${ }^{13}$.

The study's limitations included the small sample size, while validity and reliability; for example, convergent validity, construct validity, inter-rater reliability or test-retest reliability were not examined. Future studies should examine these psychometric properties.

\section{Conclusion}

SAPS-Thai provides a comprehensive measurement of positive symptoms. It indicated validity of content and known-group, and satisfactory internal consistency. It is recommended that further research should be undertaken to continue the analysis using more sophisticated techniques and a more robust sample size.

\section{Acknowledgment}

The author thanks for Keerati Pattanaseri, MD, MSc Tiraya Lerhattasilp, MD, Winitra Nuallaong, MD, MSc, Papon Laohawiraphab MD, and Veevarin Charoenporn, MD for the translation and development of the SAPS-Thai. The author also would like to thank Prof. Nancy C Andreasen, MD, PhD for giving permission to translate the SAPS.

\section{Disclosure}

The author has no potential conflicts of interest to disclose.

\section{References}

1. American Psychiatric Association. Diagnostic and Statistical Manual of Mental Disorders. Washington, DC: American Psychiatric Publishing; 2013.

2. Andreasen NC. Scale for the assessment of positive symptoms (SAPS). Iowa: University of Iowa; 1984.

3. Phillips MR, Xiong W, Wang R, Gao Y, Wang X, Zhang N. Reliability and validity of the Chinese versions of the Scales for Assessment of Positive and Negative Symptoms. Acta Psychiatr Scand. 1991;84(4):364-70.

4. Peralta V, Cuesta MJ. Dimensional structure of psychotic symptoms: an item-level analysis of SAPS and SANS symptoms in psychotic disorders. Schizophr Res. 1999;38(1):13-26.

5. Beaton DE, Bombardier C, Guillemin F, Ferraz MB. Guidelines for the process of cross-cultural adaptation of self-report measures. Spine (Phila Pa 1976). 2000;25(24):3186-91.

6. Borsa JC, Damásio BF, Bandeira DR. Cross-cultural adaptation and validation of psychological instruments: some considerations. Paidéia (Ribeirão Preto). 2012;22(53):423-32.

7. Lynn MR. Determination and quantification of content validity. Nurs Res. 1986;35(6):382-5.

8. Polit DF, Beck CT. The content validity index: are you sure you know what's being reported? Critique and recommendations. Res Nurs Health. 2006;29(5):489-97.

9. Charernboon T, Patumanond J. Social Cognition in Schizophrenia. Ment Illn. 2017;9(1):7054.

10. Mueser KT, Sayers SL, Schooler NR, Mance RM, Haas GL. A multisite investigation of the reliability of the Scale for the Assessment of Negative Symptoms. Am J Psychiatry. 1994;151(10):1453-62.

11. Daniel DG. Issues in selection of instruments to measure negative symptoms. Schizophr Res. 2013;150(2-3):343-5.

12. Andreasen NC. Methods for assessing positive and negative symptoms Mod Probl Pharmacopsychiatry. 1990;24:73-88.

13. Streiner DL. Starting at the beginning: an introduction to coefficient alpha and internal consistency. J Pers Assess. 2003;80(1):99-103. 